\title{
Stable oscillating nonlinear beams in square-wave-biased photorefractives
}

\author{
Giorgio Maria Tosi-Beleffi, Marco Presi, and Eugenio DelRe \\ Fondazione Ugo Bordoni, Via B. Castiglioni 59, 00142 Rome, Italy, Istituto Nazionale Fisica della Materia, \\ Unità di Roma 1, 00100 Rome, Italy, and Dipartimento di Fisica, Università Roma Tre, 00146 Rome, Italy
}

\section{Danilo Boschi and Claudio Palma}

Dipartimento di Fisica, Università Roma Tre, 00146 Rome, Italy, and Istituto Nazionale Fisica della Materia, Unità di Roma 3, Rome, Italy

\author{
Aharon J. Agranat \\ Department of Applied Physics, Hebrew University of Jerusalem, Jerusalem 91904, Israel
}

Received June 21, 2000

\begin{abstract}
We demonstrate experimentally that, in a paraelectric, nonstationary boundary conditions can dynamically halt the intrinsic instability of quasi-steady-state photorefractive self-trapping, driving beam evolution into a stable oscillating two-soliton-state configuration. (C) 2000 Optical Society of America

OCIS codes: $190.5330,230.3090$.
\end{abstract}

The propagation of light in a photorefractive crystal gives rise to intense beam self-action that, in its most generic manifestation, causes fanning and anisotropic scattering. ${ }^{1}$ The application of an external-bias field to the crystal can drastically change this behavior and allow spatial self-trapping and soliton formation, i.e., nondiffracting propagation of micrometer-sized optical beams. $^{2}$ In a biased system this occurs only in a transient regime, for a finite time window, and the resulting nonlinear waves are called quasi-steady-state solitons. ${ }^{3}$ Increasing the natural dark change conductivity can make this regime stable, giving rise to steady-state screening solitons. ${ }^{4}$

In this Letter we investigate, for what is believed to be the first time, a fundamentally different stabilization process from that described above, i.e., a process connected to beam behavior in a nonstationary external-bias field. ${ }^{5}$ In particular, we study beam evolution in the presence of an alternating field in centrosymmetric potassium lithium tantalate niobate (KLTN), ${ }^{6}$ a material known to support a rich variety of nonlinear beam phenomena. ${ }^{7-10}$

Results indicate that for appropriate conditions the beam self-trapping process, which leads to transient quasi-steady-state solitons for stationary conditions, can be driven into a stable self-trapped regime formed by an alternating oscillation between two beam trajectories, in the absence of enhanced dark conductivity. This phenomenon, in our understanding, is made possible by the fact that single optical trajectories, which correspond to the two alternate states of the bias field and are noncoincident owing to asymmetric diffusion-seeded bending and electro-optic readout effects, engender the simultaneous formation, through the quadratic electro-optic response of the crystal in the paraelectric phase, of two trapping index patterns. These patterns form two back-to-back specular double layers of charge that halt runway charge buildup.

Experiments are carried out in samples of zero-cut centrosymmetric photorefractive KLTN, a composite perovskite doped with copper and vanadium impurities, with a setup that is similar to those generally used in photorefractive soliton studies, ${ }^{7,8}$ apart from the absence of background illumination and the use of an alternating external voltage source. The sample temperature is kept at a given value $T$ by means of a stabilized current-controlled Peltier junction. A $\lambda=514 \mathrm{~nm}$ cw TEM $_{00}$ beam from an argon-ion laser is first expanded and then focused on the input facet of the sample and is then launched along the crystal's principal axis $z$. Focusing is obtained with either a cylindrical $y$-oriented lens, giving rise to a onedimensional beam confined in the $x$ transverse direction, for investigation of slab solitons, or a spherical lens for full two-transverse-dimensional (i.e., $x$ and $y$ ) investigation of needle solitons. The electrodes are deposited on the $x$ facets, and the source can provide a square-voltage waveform of variable peak-to-peak
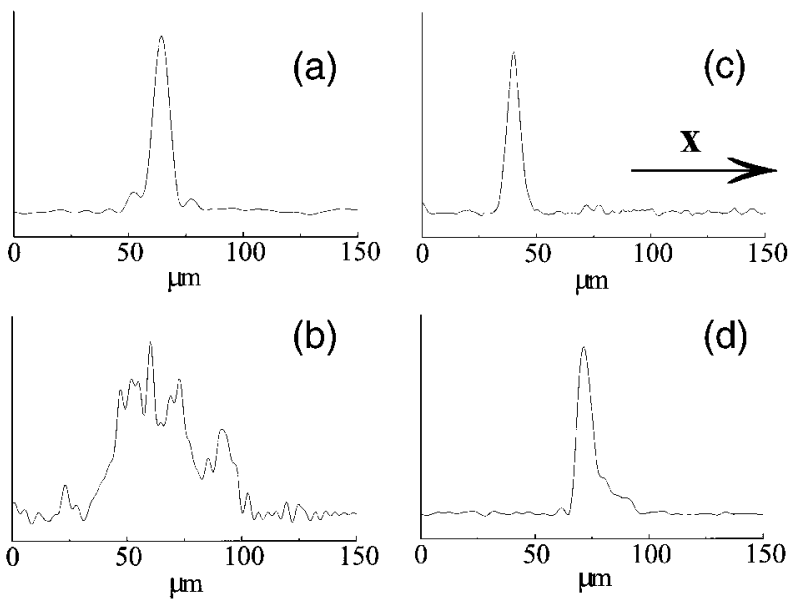

Fig. 1. Stable two-state oscillation of a slab-soliton beam subject to a square-wave bias: (a) normalized input intensity profile; (b) output intensity profile after 2.4-mm linear propagation in the crystal; (c), (d) output intensity self-trapped profiles of the two alternating states. 
amplitude $V_{\mathrm{sq}}$ and period $T_{\mathrm{sq}}$. The beam dynamics are observed from a top view and simultaneously on the transverse plane by use of two CCD cameras.

The main qualitative phenomenology observed is shown in Fig. 1, in which the two-branch oscillation along the $x$ axis is shown. The laser beam is focused by means of an $f=150 \mathrm{~mm}$ cylindrical lens onto the input facet of a $3.7^{(x)} \mathrm{mm} \times 4.7^{(y)} \mathrm{mm} \times 2.4^{(z)} \mathrm{mm}$ sample, giving rise to an approximately onedimensional (transverse dimension $x$ ) fundamental Gaussian beam diffracting in the $x$ direction, as the beam evolves along $z$. The input beam has a FWHM of $\simeq 5 \mu \mathrm{m}$ and is diffracted to $44 \mu \mathrm{m}$ after propagating $2.4 \mathrm{~mm}$ in the sample $(n \simeq 2.4)$ [see Fig. 1(a) and 1(b)]. The beam has a peak intensity $I_{p} \simeq 3 \mathrm{~kW} / \mathrm{m}^{2}$, and no background illumination is implemented. In these conditions, but with a stationary bias, quasi-steady-state self-trapping is observed after a response time $\tau_{1} \simeq 3 \mathrm{~min}$, for an external voltage on the $x$ electrodes of $V=380 \mathrm{~V}$. In the oscillating configuration of Figs. 1(c) and 1(d), the external square-wave bias voltage has a peak-to-peak amplitude $V_{\mathrm{sq}}=760 \mathrm{~V}$ with a period $T_{\mathrm{sq}}=10 \mathrm{~s}$ (i.e., $T_{\mathrm{sq}} \ll \tau_{1}$ ), and the crystal is kept at $T=18^{\circ} \mathrm{C}$ (thus it has a relative dielectric constant $\epsilon_{r} \approx 11 \times 10^{3}$ ). As external oscillation occurs, the beam undergoes spatial confinement in one direction toward one electrode [Fig. 1(c)], and then in the other, toward the opposite electrode [along the transverse $x$ direction; Fig. 1(d)], oscillating between two distinct optical trajectories. The remarkable result is that this stable oscillating regime, which has a buildup transient $\tau_{2} \simeq 15 \mathrm{~min}$, considerably longer than $\tau_{1}$, continues indefinitely and is thus a stable oscillation in which the beam is continuously confined but in two distinct alternating trajectories. Switching time $\tau_{\mathrm{sw}} \ll T_{\mathrm{sq}}$ is associated with the crystal charging time. Apart from this very short interval $\tau_{\mathrm{sw}}$, which is not photorefractive in nature, the beam is continuously trapped. The distance between the two trapped beams at the output is $\Delta x \simeq 30 \mu \mathrm{m}$.

An analogous phenomenology is observed for twodimensional diffracting beams and is shown in Fig. 2, in a second $2.2^{(x)} \times 2.2^{(y)} \times 6.4^{(z)}$ sample of KLTN kept at $T=26{ }^{\circ} \mathrm{C}$ (see Ref. 8 for details on the crystal). Note that the needle confinement extends over approximately 25 diffraction lengths.

As opposed to the case of screening soliton phenomena, here we found no strict existence condition associated with the electro-optic response (i.e., $V_{\mathrm{sq}}$ and crystal $T$ ), much as in standard quasi-steady-state self-trapping experiments. ${ }^{3}$ The only observable difference in the final oscillating state that depends appreciably on changes in the electro-optic response is the divergence angle of the two trajectories, which gives rise to a different value of $\Delta x$ at the output. We found that a stronger static polarization induces a stronger divergence. As we show below, this behavior is connected to the electroholographic response in the paraelectric phase.

However, we observe a distinct dependence of beam evolution on the time scales involved, suggesting that the main underlying mechanism is strongly connected to the temporal oscillations in the boundary conditions. We thus carried out experiments in which we changed the nonlinear time constant connected to beam peak intensity $I_{p}$, keeping all other parameters unaltered. Thus we essentially varied the ratio $T_{\mathrm{sq}} / \tau_{1}$, since $\tau_{1}$ is approximately proportional to $I_{p}$. For slow enough dynamics, i.e., for $T_{\mathrm{sq}} / \tau_{1} \ll 1$, the steady oscillating state is always reached (at least for the investigated cases), whereas for very rapid dynamics, i.e., for beam intensities such that $T_{\mathrm{sq}} / \tau_{1} \gg 1$, single-branch evolution is allowed to reach quasi-steady-state destabilization, and steady oscillation is not observed. In this case the beam undergoes a distinctive swinging evolution that mimics the two-state self-trapping of the previous case, as shown in Fig. 3. During one field oscillation, the beam first undergoes self-trapping, deflecting in one direction [Fig. 3(b)], decays [see Fig. 3(a)], diffracting in the forward $z$ direction, forms a second transient soliton in the opposite direction [Fig. 3(c)], decays again in the $z$ direction, and so forth. Thus, in this case, no stable self-trapped oscillating configuration is reached, since most of the time the beam is diffracting $\left(T_{\mathrm{sq}} \gg \tau_{1}\right)$. Note that the swinging motion is a direct consequence of the residual charge displacement of the previous state and is much more pronounced than the single-beam self-bending observed in stationary conditions $(\Delta x \simeq 30 \mu \mathrm{m}$ as compared with the $\Delta x \simeq 5 \mu \mathrm{m}$ conventional selfbending that was observed; see Fig. 1).
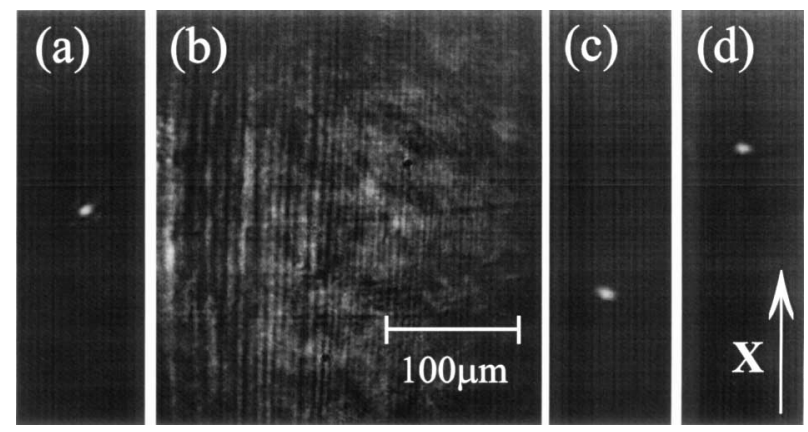

Fig. 2. Stable two-state oscillation of a needle-soliton beam subject to a square wave of peak-to-peak amplitude $V_{\mathrm{sq}}=700 \mathrm{~V}$ and period $T_{\mathrm{sq}}=10 \mathrm{~s}$ : (a) input intensity 6- $\mu \mathrm{m}$ FWHM distribution; (b) output intensity $150-\mu \mathrm{m}$ distribution after $6.4-\mathrm{mm}$ linear propagation in the crystal; (c), (d) output intensity self-trapped profiles of the two alternating states.

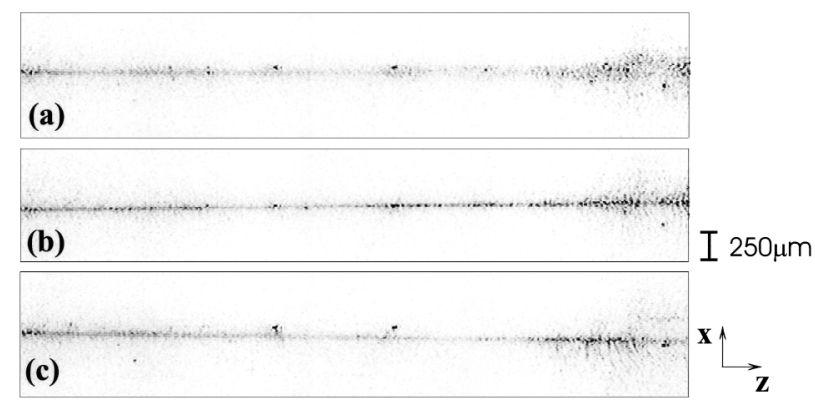

Fig. 3. Soliton swinging: slab-beam transient dynamics for $\tau_{1} \simeq 2 \mathrm{~s}$ and $T_{\mathrm{sq}}=10 \mathrm{~s}$ in a $6.4-\mathrm{mm}$-long sample of KLTN. (a) Top view ( $y$ direction) of linear diffraction. (b), (c) Two opposite transient self-trapped states. 


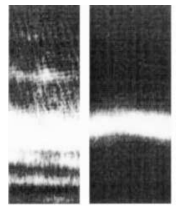

(a) (b)

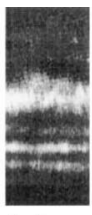

(c)

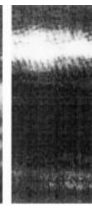

(d)

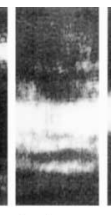

(e) (f)

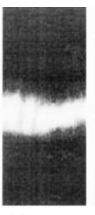

(g)

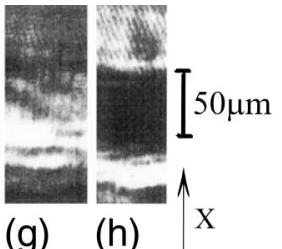

(h)
Fig. 4. Breakdown of the two-state formation process, with the output intensity distribution conditions of Fig. 1: (a) initial output diffraction for $V=0$, (b) quasisteady-state self-trapping for $V=V_{+}$, (c) readout intensity for $V=0$ (after soliton formation), (d) readout intensity for $V=V_{-}$, (e)-(h) specular results starting from $V=V_{-}$.

We limit our discussion to slab solitons, since even basic needle-soliton phenomenology is still unclear. ${ }^{11}$ Concerning the formation of the two-state oscillation, we note that, although in a purely driftlike configuration a free photoexcited charge under the influence of a zero-average square-wave alternating field, $E_{\mathrm{sq}}$, with $T_{\text {sq }} \ll \tau_{1}$, cannot give rise to any space-charge separation (unlike for sillenites ${ }^{12}$ ), asymmetric-charge diffusion components can seed two intensity distributions, $I_{+}$and $I_{-}$. These distributions correspond to the two alternate states of the external field, which separate during propagation along the $z$ axis, and this allows a nonzero photorefractive response. ${ }^{13}$ The final state is a product of beam-charge separation during one electric field polarity, combined with electroholographic effects during the opposite-polarity phase. ${ }^{14}$

To break down the process, we consider the formation of a single quasi-steady-state soliton with a constant voltage $V=V_{+}$(equal to the positive value of the alternating field in the oscillating case) [see Figs. 4(a) and 4(b)]. If we halt the beam evolution (attenuating the propagating beam intensity) before the transient trapped regime has decayed (i.e., for $t<\tau_{1}$ ) and set $V=0$, we observe increased beam diffraction at the output, which is a signature of the residual defocusing pattern [Fig. 4(c)]. Diffraction is furthermore slightly asymmetric as a consequence of the diffusion component in the charge separation. If we invert the applied electric field (i.e., $V=V_{-} \equiv-V_{+}$), the defocusing pattern is enhanced, and most of the light is diverted to a limited region that is strongly shifted with respect to the input beam [Fig. 4(d)]. Wholly specular behavior is observed when the quasi-steady-state soliton is originally formed with $V=V_{-}$[Figs. 4(e)-4(h)]. In the actual alternating-field case, since we start from a zero charge separation and do not allow the system to evolve, if not only partially, during each oscillation $\left(T_{\mathrm{sq}} \ll \tau_{1}\right)$, the final charge separation and index pattern will be a symmetric hybrid combination of the two self-trapped and two deflected beams [see Figs. 4(b), 4(d), 4(f), and 4(h)], whereas the actual beam trajectory will switch, following the oscillation of the external field. Note that during the entire oscillation the beam continuously maintains its confinement, apart from the small transient $\tau_{\mathrm{sw}}$.
Concerning the stability of the nondiffracting pattern, in the absence of artificial dark illumination, we note that in our case charge separation is intrinsically symmetric. Mobile electrons (impurities form mostly donor sites in KLTN) are forced to drift toward the central region between the two trajectories, forming a potential barrier to further charge separation and saturation, effectively freezing the space-charge structure. The switching in this case is only electro-optic in nature and occurs as a consequence of the electroholographic readout of the asymmetric index components in the initial stages of propagation (where the two trajectories are superimposed) after the space-charge field has reached a steady state.

The work of G. M. Tosi-Beleffi, M. Presi, and E. DelRe was carried out within the framework of an agreement between Fondazione Ugo Bordoni and the Italian Communications Administration. Research carried out by A. J. Agranat was supported by a grant from the Ministry of Science of the State of Israel. We thank Luigi Piccari for useful discussions. E. DelRe's e-mail address is edelre@fub.it.

\section{References}

1. L. Solymar, D. J. Webb, and A. Grunnet-Jepsen, The Physics and Applications of Photorefractive Materials (Clarendon, Oxford, 1996).

2. M. Segev and M. Stegeman, Phys. Today 51(8), 42 (1998); G. I. Stegeman and M. Segev, Science 286, 1518 (1999).

3. G. C. Duree, J. L. Shultz, G. J. Salamo, M. Segev, A. Yariv, B. Crosignani, P. Di Porto, E. J. Sharp, and R. R. Neurgaonkar, Phys. Rev. Lett. 71, 533 (1993).

4. M. Segev, G. Valley, B. Crosignani, P. Di Porto, and A. Yariv, Phys. Rev. Lett. 73, 3211 (1994); D. N. Christodoulides and M. I. Carvalho, J. Opt. Soc. Am. B 12, 1628 (1995).

5. In linear configurations; see, e.g., A. Grunnet-Jepsen, C. H. Kwak, and L. Solymar, Opt. Lett. 19, 1299 (1994).

6. A. J. Agranat, R. Hofmeister, and A. Yariv, Opt. Lett. 17, 713 (1992).

7. E. DelRe, B. Crosignani, M. Tamburrini, M. Segev, M. Mitchell, E. Refaeli, and A. J. Agranat, Opt. Lett. 23, 421 (1998).

8. E. DelRe, M. Tamburrini, M. Segev, E. Refaeli, and A. J. Agranat, Appl. Phys. Lett. 73, 16 (1998).

9. B. Crosignani, A. Degasperis, E. DelRe, P. Di Porto, and A. J. Agranat, Phys. Rev. Lett. 82, 1664 (1999).

10. E. DelRe, M. Tamburrini, M. Segev, R. Della Pergola, and A. J. Agranat, Phys. Rev. Lett. 83, 1954 (1999).

11. S. Gatz and J. Herrmann, Opt. Lett. 23, 1176 (1998); M. Saffman and A. A. Zozulya, Opt. Lett. 23, 1579 (1998).

12. P. A. Marquez Aguilar, J. J. Sanchez Mondragon, S. Stepanov, and V. Vysloukh, Phys. Rev. A 54, R2563 (1996).

13. M. Carvalho, S. Singh, and D. Christodoulides, Opt. Commun. 120, 311 (1995).

14. E. DelRe, M. Tamburrini, and A. J. Agranat, Opt. Lett. 25, 963 (2000). 